DOI: 10.17234/SRAZ.65.22

\title{
La littérature française dans la bande dessinée croate
}

\author{
Patrick Levačić \\ Luka Baule \\ Université de Zadar \\ plevacic@unizd.hr \\ lukabaule1@gmail.com
}

Le but de cet article est de rechercher pourquoi, comment et de quelle façon la littérature française a été transposée en bande dessinée (dorénavant B.D.) croate. L'idée est d'introduire dans une étude interculturelle générale un champ de recherche intermédial. La naissance du neuvième art croate est étroitement liée à la littérature française. Le dessinateur Andrija Maurović et le scénariste Krešimir Kovačić ont adapté en 1935 le roman de Paul Féval, fils, Mam'zelle Flamberge (Vjerenica mača). Le dessinateur Ferdo Bis et le scénariste Marcel Čukli vont créer entre 1940-1942 l'adaptation du roman Atlantide de Pierre Benoit. La B.D. sera ensuite publiée dans l'hebdomadaire Zabavnik de 1943 jusqu' en 1945. Ces adaptations sont-elles fidèles au roman français?

Mots-clés : Paul Féval, fils, Mam'zelle Flamberge, Pierre Benoit, Atlantide, B.D. croate

\section{Introduction}

Selon des recherches menées jusqu'à présent on peut constater que la littérature française n'est qu'à peine mentionnée dans la bande dessinée croate, et cela d'abord dans le contexte du développement historique. Notre première préoccupation était de trouver une trace de toutes les œuvres françaises dans la bande dessinée croate. Dans la conclusion de Hroatski strip 1945-54 il y a deux romans adaptés de Jules Verne : Vingt Mille Lieues sous les mers et De la Terre à la Lune. Ce dernier est lié au roman de H.G. Wells Les premiers Hommes dans la Lune (Macan 2007: 41). La B.D. de Maurović, Opsada (Le Siège de Zadar) datant de 1951, n'entrerait pas dans cette sélection bien qu'il existerait peut-être une liaison avec les chroniques françaises du Moyen Âge. Dans ce travail on se limite à deux romans, Mam'zelle Flamberge (1911) et Atlantide (1919), que nous considérons importants pour l'analyse de la relation entre la littérature française et la B.D. croate. 


\section{Mam'zelle Flamberge et le jour de la naissance du $9^{\text {ème }}$ art croate}

L'adaptation du roman français de Paul Henri Féval fils (1860-1933) Mam'zelle Flamberge, était dessinée par le père de la B.D. croate Andrija Maurović tandis que l'adaptation scénaristique était réalisée par Krešimir Kovačić. Dans Novosti de Zagreb du 12 mai 1935 est apparue l'annonce d'un type spécial du roman connu sous l'appellation de «roman en images», «un film journalistique sensationnel « et «un film journalistique à suspens», ce qui représente en même temps la naissance du $9^{\text {ème }}$ art croate (Aljinović 2012: 82). Maurović, dans la conversation avec Kovačić n'utilise pas non plus la notion de la bande dessinée, mais de «roman en images» (Vojinović 2007:55). Du point du vue historique, la B.D. comme un nouveau media chercherait sa légitimité à travers la littérature (Peeters 2003:105) où, dans le contexte croate, à travers l'art cinématographique, déjà affirmé et culturellement valorisé par Vera Horvat-Pintarić (Maurović 1983: 8).

Le scénariste Krešimir Kovačić était à Paris de 1928 jusqu'à 1935 comme correspondant permanant de Novosti. Sa nouvelle rencontre en 1935 avec Maurović serait déterminante. En venant à Zagreb, Maurović l'accueille à la rédaction de Novosti avec les mots suivants: «J'ai parcouru depuis longtemps toute la littérature croate, mais aucun de ces ouvrages n'était assez adapté pour un 'roman en images', même pas celui de ton père, U registraturi. Ça doit être un contenu vivant ... d'un suspens comme dans un film et que ça vaut la peine d'être illustré». (Vojinović 2007:56). Kovačić, qui était un bon connaisseur de la littérature française, s'est décidé pour le roman historique aventurier de Paul Henri Féval fils. L'histoire se situe au $18^{\text {ème }}$ siècle et dépeint la vie des châteaux. C'est un environnement social dans lequel l'honneur personnel et la loyauté envers le Roi et la patrie sont appréciés comme les plus hautes valeurs. Les héros habitués aux épées qui se battent bravement pour la justice. Mais, c'est aussi l'époque des intrigues, des complots et des mensonges. Dans ce tourbillon dangereux apparait l'héroïne principale du roman Mam'zelle Flamberge, la jeune Henriette, la fille du comte Lespare. Déguisée en homme, elle lutte avec les autres mousquetaires. Quand les aventures se terminent, elle va laisser son épée et devenir la fiancée du messager royal, le comte Jacques de Courten (Aljinović 2012: 81). Kovačić a choisi le roman qui correspondait aux préférences de Maurović. C'est une histoire d'héroïsme et d'aventures dans laquelle la justice doit prévaloir. Selon les souvenirs de Maurović, Kovačić lui apportait le plus souvent le matériel pour trois, quatre jours d'avance (Aljinović 2012: 83). Le processus exact de la création qui s'est déroulé entre Maurović et Kovačić n'est pas entièrement éclairé, mais chaque adaptation d'une œuvre littéraire en B.D. est un procédé sémiologique entre le texte et l'image dans lequel il est nécessaire de réduire le nombre de personnages en mettant l'accent que sur le déroulement principal de l'histoire. (Kadima-Nzuji 2001:55). Maurović a réussi, par un cadrage cinématographique, à dynamiser le roman et à augmenter l'effet dramatique de certaines scènes (Aljinović 2012: 83). L'adaptation d'un roman pour la B.D. ne doit pas signifier que la littérature serait perdante, mais au contraire, à travers le $9^{\text {ème }}$ art, elle peut mieux mettre en scène certaines figures du style. L'analepse et l'ellipse dans cette B.D. ont une valeur 
fonctionnelle et esthétique dans la formation de la structure narrative. Maurović et Kovačić l'ont affirmé, par le biais des effets dramatiques et figures stylistiques. Dans le roman Mam'zelle Flamberge, la culmination dramaturgique et l'intrigue principale se perpétuent pendant la découverte de l'identité réelle du Gonsalvo, le personnage négatif. Il est le traitre qui se cache habillement derrière le Roi Louis $\mathrm{XV}$, qui lui est favorable. Tortillard, un personnage camouflé comme un bossu, en réalité le mari de la comtesse Constance et le père d'Henriette, va persuader Gonsalvo de rencontrer la comtesse. Gonsalvo va prier Constance d'accepter son amour car avec le mariage, il espère s'approprier ses biens (Féval 1950, 23-24, 145-146). Il insiste à maintes reprises. Elle l'éconduit, alors il se sent humilié et ses intentions cachées se révèlent au grand jour (les vignettes 197-201), (Maurović/ Kovačić 2006: 24-25). Maurovic reflète parfaitement l'ambiance du roman par sa façon de cadrer les séquences et de valoriser la scénographie. Dans ce contexte les vignettes de 197-201 sont fidèles au roman:

Je vous aime!... Consentez à accepter mon amour et mon nom, et votre fils vous sera rendu. Jamais ! Jamais ! hurla Henriot ${ }^{1}$ en commençant à se redémener comme un furieux. Jamais! répéta la comtesse retrouvant sa hautaine froideur. Qu'il soit donc fait selon votre volonté, madame. (Féval 1950: 145)

Gonsalvo insistera par la suite encore plus :

Je vous aime! Je vous aime comme un fou! Comme ami du roi, je puis vous faire la femme la plus enviée de France. Je puis vous rendre au centuple le bonheur que vous croyez avoir perdu ... Non? Vous refusez!... vous refusez toujours!... Ah! Femme orgueilleuse et entêtée que vous êtes!... Vous serez perdue quand même! Et vous l'êtes déjà!... (Féval 1950: 145).

Après cela apparait l'attitude protectrice d'Henriette/Henriot:

Ma mère, lança Henriot avec une énergie admirable, relevez-vous!... Ne vous humiliez point devant les assassins de mon père! (Féval 1950: 146)

Tortillard (le père déguisé d'Henriot/ Henriette) apparait soudainement et miraculeusement comme un sauveur ou en deus ex machina:

L'arrivée inopinée d'un nouvel acteur venait d'opérer ce miracle. Il n'était ni bien bâti ni fort, selon l'apparence, ce personnage dont l'entrée produisait un aussi contrariant effet, et pourtant il venait de se poser en deus ex machina en passant entre Henriot et Piétri avec une si maladroite brutalité que le stylet de ce dernier avait échappé à sa main. (Féval 1950, 146)

De la perspective d'aujourd'hui, la vignette 200 (Maurović / Kovačić 2006: 25) contient trop d'informations à cause du stylet mentionné dans le texte et dans

1 Henriette déguisée. 
l'image. Il faut garder à l'esprit que pour les lecteurs d'antan ce n'était pas trop car il fallait susciter l'émotion. La présence du stylet est donc justifiée. C'est un cadre qui démontre un démasquage crucial du personnage négatif. Tortillard/ le bossu prétend reconnaitre qu'il entretenait une liaison amoureuse avec la comtesse Constance. Gonsalvo acceptera cela car il croit pouvoir, malgré tout, faire du chantage à la comtesse. La situation se décante lorsque Tortillard, révèle que c'est lui, en fait, le mari de la comtesse (Maurović/Kovačić 2006: 29). L'exemple mentionné est intéressant dans une perspective intermédiale et interculturelle de la relation entre la littérature et la B.D. Par le biais de la B.D. croate on découvre le roman français, cependant en 1935, c'est l'inverse que l'on observait : c'est via le roman, que le chemin vers un nouveau media artistique était recherché.

On constate que certains romans français ont été découverts par les lecteurs croates que via la B.D. car ils n'ont pas étaient traduits en croate. C'est le cas aujourd'hui avec le roman Ja, François Villon de Jean Teulé (édition Fibra 2018), et c'était le cas jadis avec Mam'zelle Flamberge, le roman de Féval, P. fils. Les adaptations doivent permettre aux lecteurs de se représenter de manière fidèle le récit adapté. Cependant, il serait bien dommage de substituer l'œuvre originale par son adaptation en B.D.

\section{Atlantide de Pierre Benoit dans la B.D. croate}

Selon la critique littéraire, il s'agit du roman colonial (Moretti 1998: 59) dont l'action se déroule au nord de l'Afrique. La notion de l'Atlantide peut avoir aussi une connotation géographique liée à la politique coloniale de la France :

Peupler au plus vite, à tout prix, cette colonie de peuplement, voilà la tâche primordiale qui s'impose à la Vieille France. L'Atlantide doit devenir la France Nouvelle (Reclus 1919: IX).

D’un autre côté, la création du roman est motivée différemment. L'idée du fond est la disparition mystérieuse d'un explorateur français, c'est ce qui a marqué l'enfance de l'auteur passé en Tunisie et en Algérie :

De 1892 à 1907, j’ai vécu en Tunisie et en Algérie. Dès mon enfance, j’ai entendu parler des Touareg, et mon imagination a été excitée par certaines sombres histoires, celle notamment d'une mission exécutée dans le centre africain par deux Français, dont un seul des deux explorateurs était revenu, sans qu'on ait pu jamais savoir exactement comment avait péri son compagnon. Telle est l'idée qui est à la base de l'Atlantide. Il n'y en a pas d'autre (Benoit 1920:1).

Le mythe de l'Atlantide se réalise à travers un aspect mystique du désert. Les explorateurs ou les officiers français n'arrivent pas à atteindre l'Atlantide. En effet, chez la reine Antinéa ils seront enivrés par sa beauté et après avoir goûtés à son amour, ils meurent paisiblement. Le roman est une histoire d'amour qui met en évidence le mythe de l'Atlantide au féminin (Foucrier, 2004: 103), elle incarne une femme fatale: 
Une femme s'est rencontrée pour rétablir au profit de son sexe la grande loi hégélienne des oscillations. Séparée du monde aryen par la formidable précaution de Neptune, elle évoque vers elle les hommes les plus jeunes et les plus vaillants. (...) Elle leur prête son corps tandis qu'elle les domine de son âme (Benoit 1928: 151).

Son caractère sadique et un comportement envers les personnages masculins confirme l'idée que «le meurtre est le dernier excès de la volupté» (Sade, 1969 : 213). Antinéa symbolise Éros et Thanatos:

Elle vient, une fois par jour, dans cet hypogée. Elle s'arrête devant ces stalles. Elle touche ces poitrines froides, qu'elle a connues si brûlantes. Puis, après avoir rêvé autour de la stalle vide où bientôt il dormira pour toujours dans sa froide gaine d'orichalque, nonchalante, elle s'en retourne vers celui qui l'attend (Benoit 1928: 151).

L'adaptation croate souligne aussi la femme fatale ou Éros et Thanatos, avec la différence essentielle que dans la B.D. cela apparait avec moins d'intensité. Dans le roman Antinéa va troubler les officiers français : «Famille, patrie, honneur, tout, vous renierez tout pour elle» (Benoit 1928:152). Dans la B.D. cela sera exclu. On peut trouver trois raisons à cela. Premièrement, dans la période de L'Etat indépendant de Croatie (1941-1945) l'idée de la supériorité féminine, n'était pas acceptable bien que l'hebdomadaire Zabavnik avait idéologiquement résisté. Deuxièmement, les personnages masculins positifs dans la B.D croate sont particulièrement visibles lorsque le contenu principal est lié à l'amour envers la patrie ; un bon exemple est le personnage de Radoslav dans la B.D. Seoba Hrvata $\mathcal{E}$ Knez Radoslav de Maurović. Troisièmement le visuel durant la guerre a été formé autour de la relation femme/soldat afin de servir à renforcer leur moral (Horvat 2019: 248), et nullement pour questionner l'idée de l'amour pour la patrie.

\section{Conclusion}

Pour l'adaptation de Mam'zelle Flamberge l'intérêt de Krešimir Kovačić pour la littérature française et son séjour à Paris étaient déterminants. Il avait choisi le roman dont le genre et le contenu correspondaient intimement au style de Maurović, comme c'est le cas avec cette histoire héroïque et aventurière dans laquelle la justice emporte face à l'hypocrisie qui règne à la vie de cour. Il est évident que depuis la rencontre avec Kovačić, Maurović savait très bien ce qui pouvait plaire au public, et la réussite était au rendez-vous car, entre autre, il présentait la bande dessinée comme quelque chose de nouveau (roman en images, narration cinématographique). Pour l'adaptation de l'Atlantide il n'y avait pas d'intérêt direct des auteurs pour la littérature française. Les auteurs Ferdo Bis i Marcel Čukli ont probablement utilisé la traduction croate de l'Atlantide de l'année 1921. Le monde mystique, un caractère de suspens et d'amusement de la B.D. et la relation entre les officiers français et l'irrésistible Antinéa sont des thèmes appréciés qui détournent l'attention des lecteurs des tensions sociales provoqués par la guerre. Pour l'adaptation de l'Atlantide, à la différence de 
Mam'zelle Flamberge, les circonstances sociales étaient plus importantes que les intérêts des auteurs.

L'adaptation de l'Atlantide n'est pas liée au discours colonial. Le roman combine la fiction et les sources historiques et tend à créer chez le lecteur une impression de vraisemblance, ce qui n'est pas le cas pour la B.D., orientée vers le divertissement. Le thème du monde mystique du désert où disparaissent les explorateurs, accapare l'imaginaire du lecteur. Antinéa réunit l'aspect de la femme fatale, Éros et Thanatos, ce qui aussi correspond à la B.D. mais ne fait pas oublier la patrie comme c'est le cas dans le roman.

\section{Références bibliographiques}

Aljinović, Rudi (2012). Kad je strip bio mlad: prilozi za povijest hrvatskog stripa. Zagreb: Stripforum.

Benoit, Pierre (1920). Comment j’ai écrit l'Atlantide, in : L'Écho de Paris, n¹2592, 02/02, pp.1-2.

Benoit, Pierre (1928). L'Atlantide, Coulommiers: Librairie Hachette.

Féval, P. fils. (1950). Mam'zelle Flamberge. Paris: Librairie Arthème Fayard.

Foucrier, Chantal (2004). Le mythe de l'Atlantide (1800-1939). Grenoble: ULLUG.

Horvat, Dina (2019). Andrija Maurović i hrvatski strip (1901.-1981.), Pro tempore, n¹4, pp. 234-264. https://hrcak.srce.hr/index.php?show=clanak\&id_clanak_jezik=338120

Kadima-Nzuji, Mukala (2001). L'adaptation de textes naratives à la bande dessinée: le cas congolais, Revue des littératures du Sud. Paris: Notre Librairie. pp.5257. https://gallica.bnf.fr/ark:/12148/bpt6k6494759x/f57.image.r=bande $\% 20$ dessinee

Macan, Darko (2007). Hrvatski strip 1945.-54. Zagreb: Mentor.

Maurović, Andrija (1983). Andrija Maurović, Biser Zla, Ukleti brod...(préf. Darko Glavan). Niš:Prosveta.

Maurović, Andrija / Kovačić Krešimir (2006). Vjerenica mača. Format: Osijek

Moretti, Franco (1998). Atlas of the European Novel, (1800-1900). London-New York: Verso.

Peeters, Benoît (2003). Lire la bande dessinée, Barcelone: Flamamarion.

Reclus, Onésime (1919). L'Atlantide, pays de l'Atlas: Algérie, Maroc, Tunisie (préf. Paul Pelet). Paris:La Renaissance du livre.

Sade, Marquis (1969). Les prosperités du vice. Paris : Union générale d'éditions. 


\section{Francuska književnost u hrvatskom stripu}

Cilj rada je istražiti kako i na koji način se francuska književnost adaptirala u hrvatski strip. Rođenje hrvatske devete umjetnosti pojavljuje se 1935.godine preko adaptacije romana Mam'zelle Flamberge (Vjerenica mača) Paula Févala sina (1860-1933). Autori su Andrija Maurović i scenarist Krešimir Kovačić. U tjedniku Zabavnik se od 1943. do 1945. pojavljuje strip Atlantida što je adaptacija francuskog romana Atlantide (1919) autora Pierrea Benoita (1886.-1962.). Hrvatski autori su Ferdo Bis i Marcel Čukli. Obje adaptacije vjerodostojno su prenijele glavni sadržaj, dramski sukob i poruku s izvornih predložaka. Razlike postoje na razini da se adaptacije trebaju funkcionalno i tematski prilagoditi najprije hrvatskom čitatelju pa se u tom smislu gubi vjerodostojnost samih predložaka. U Atlantide imamo kolonijalne motive i aspekt femme fatale koja može i domoljublje staviti u sjenu, što nisu teme za adaptaciju stripa iz ideoloških razloga. Taj roman se adaptirao preko hrvatskog prijevoda iz 1921.godine što je različito od stripa Vjerenica mača kojega je adaptirao poznavatelj francuske književnosti Krešimir Kovačić.

Ključne riječi: Paul Féval, sin, Vjerenica mača, Pierre Benoit, Atlantida, hrvatski strip 
